\title{
Contributions to integral nuclear data in ICSBEP and IRPhEP since ND2016
}

\author{
John Darrell Bess ${ }^{1, *}$, Tatiana Ivanova ${ }^{2, *}$, and J. Blair Briggs ${ }^{1, * * *}$ \\ ${ }^{1}$ Idaho National Laboratory \\ ${ }^{2}$ OECD NEA
}

\begin{abstract}
The contributions to the International Criticality Safety Benchmark Evaluation Project (ICSBEP) and the International Reactor Physics Experiment Evaluation Project (IRPhEP) was last presented to the international nuclear data community at ND2016. Since ND2016, integral benchmark data that are available for nuclear data testing has continued to increase. The 2018 edition of the International Handbook of Evaluated Criticality Safety Benchmark Experiments (ICSBEP Handbook) now contains 574 evaluations with benchmark specifications for 4,916 critical, near-critical, or subcritical configurations, 45 criticality alarm placement/shielding configuration with multiple dose points apiece, and 215 configurations that have been categorized as fundamental physics measurements that are relevant to criticality safety applications. The 2018 edition of the International Handbook of Evaluated Reactor Physics Benchmark Experiments (IRPhEP Handbook) contains data from 159 different experimental series that were performed at 54 different nuclear facilities. Currently 156 of the 159 evaluations are published as approved benchmarks with the remaining three evaluations published as drafts. Measurements found in the IRPhEP Handbook include criticality, buckling and extrapolation length, spectral characteristics, reactivity effects, reactivity coefficients, kinetics, reaction-rate distributions, power distributions, isotopic compositions, and/or other miscellaneous types of measurements for various types of reactor systems. Additional benchmark evaluations will be included in the 2019 editions of these handbooks. These handbooks continue to represent the standard for neutronics benchmark experiment evaluation.
\end{abstract}

\section{Introduction}

The status of the two neutronics international benchmark projects sanctioned by the Organisation for Economic Co-operation and Development Nuclear Energy Agency (OECD NEA), the International Criticality Safety Benchmark Evaluation Project (ICSBEP) [1] and the International Reactor Physics Experiment Evaluation Project (IRPhEP) [2], was last directly discussed with the international nuclear data community at the International Conference on Nuclear Data for Science and Technology (ND2016) in Bruges, Belgium [3].

The primary purpose of the ICSBEP and IRPhEP is to provide extensively peer-reviewed integral benchmark experiment data to the international nuclear community to support validation and testing of nuclear data and models. Users of these handbooks are found throughout the nuclear data, reactor physics, criticality safety, and mathetmatics/computation communities. The contents of these handbooks support validation of analytical methodologies utilized in reactor physics, fuel cycle characterization, nuclear facility safety analysis and design, advanced modelling and simulation, training, education, and nuclear data [4].

\footnotetext{
*e-mail: john.bess@inl.gov

**e-mail: tatiana.ivanova@ oecd-nea.org

***e-mail: jblairbriggs@ @ableone.net
}

Since ND2016, the quantity of available integral benchmark data to support nuclear data testing has increased. The 2018 edition of the International Handbook of Evaluated Criticality Safety Benchmark Experiments (ICSBEP Handbook) [5] (cover shown in Fig. 1) now contains 574 evaluations with benchmark specifications for 4,916 critical, near-critical, or subcritical configurations, 45 criticality alarm placement/shielding configuration with multiple dose points apiece (contained within 7 evaluations), and 215 configurations that have been categorized as fundamental physics measurements that are relevant to criticality safety applications (contained within 8 evaluations).

The 2018 edition of the International Handbook of Evaluated Reactor Physics Benchmark Experiments (IRPhEP Handbook) [6] (cover shown in Fig. 2) contains data from 159 different experimental series that were performed at 54 different nuclear facilities. Currently 156 of the 159 evaluations are published as approved benchmarks with the remaining three evaluations published in draft format only. Measurements found in the IRPhEP Handbook include criticality, buckling and extrapolation length, spectral characteristics, reactivity effects, reactivity coefficients, kinetics, reaction-rate distributions, power distributions, isotopic compositions, and/or other miscellaneous types of measurements for various types of reactor systems. 


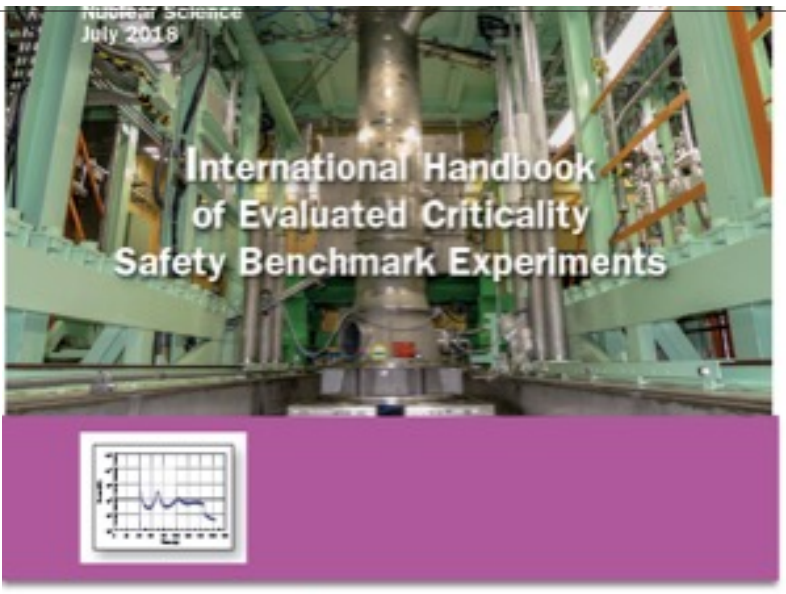

(J) NEA

Figure 1. 2018 Edition of the ICSBEP Handbook.

A total of 25 countries have contributed to the past and continued success of these projects: 22 to the ICSBEP and 20 to the IRPhEP. Contributing countries include the following: Argentina, Belgium, Brazil, Canada, People's Republic of China, Czech Republic, France, Germany, Hungary, India, Israel, Italy, Japan, Kazakhstan, Poland, Republic of Korea, Russian Federation, Serbia, Slovenia, South Africa, Spain, Sweden, Switzerland, United Kingdom, and the United States of America.

The full technical details and benchmark experiments descriptions can be located for the benchmark reports on the most recent editions of the handbooks. Many evaluations also are periodically updated as errors are identified, additional evaluated data are incorporated, or clarifications are provided based upon handbook user feedback. Handbook users are strongly encouraged to utilize the Database for the International Handbook of Evaluated Criticality Safety Benchmark Experiments (DICE) [7] and the IRPhEP Database and Analysis Tool (IDAT) [8] to verify the latest benchmark evaluation versions contained within their respective handbooks.

\section{Latest ICSBEP Contributions}

The 2018 edition of the ICSBEP Handbook is available on DVD or via direct download from the internet, and can be requested using the following website: https://www.oecd-nea.org/science/wpncs/icsbep/.

Since ND2016, the quantity of critical, subcritical, and near-critical benchmark experiment evaluations has increased by six. This includes one high-enriched uranium benchmarks, one intermediate-enriched uranium benchmarks, and four low-enriched or natural uranium benchmarks. No new plutonium, uranium-233, mixed uranium/plutonium, or other fissionable isotope benchmarks have been contributed recently. Two new criticalityalarm-placement/shielding benchmarks have been added, and one new fundamental physics benchmark. Latest contributions to the ICSBEP Handbook are summarized:
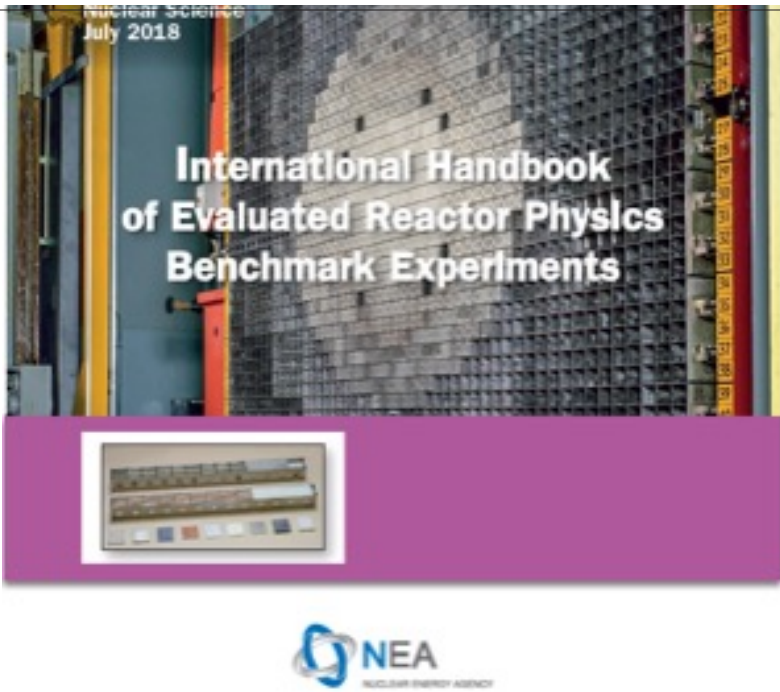

Figure 2. 2018 Edition of the IRPhEP Handbook.

\subsection{High-Enriched Uranium (HEU)}

\section{- HEU-MET-FAST-096}

Static Critical Experiments for the Sorgente Rapida (SORA) Reactor Mockup.

\subsection{Intermediate-Enriched Uranium (IEU)}

\section{- IEU-MET-FAST-024}

FCA IX-7 Assembly Experiments: 20\% Enriched Uranium Metal Core Surrounded by Depleted Uranium Metal Blanket.

\subsection{Low-Enriched Uranium (LEU)}

\section{- LEU-COMP-THERM-097}

Titanium and/or Aluminum Rod-Replacement Experiments in Fully-Reflected Water-Moderated SquarePitched U(6.90) $\mathrm{O}_{2}$ Fuel Rod Lattices with 0.67 Fuel to Water Volume Ratio $(0.800 \mathrm{~cm}$ Pitch $)$,

\section{- LEU-COMP-THERM-098}

Partially-Reflected Critical Experiment with $\mathrm{UO}_{2}-5.74$ wt.\% U-235 Fuel Rods,

\section{- LEU-COMP-THERM-100}

Lattice of U(4.378 wt.\%) $\mathrm{O}_{2}$ Rods with Polytetra Fluoroethylene Block, and

\section{- LEU-SOL-THERM-012}

TRACY: Unreflected 10\%-Enriched Uranyl Nitrate Solution in a 50-cm-Diameter Annular Tank. 


\subsection{Criticality Alarm Placement/Shielding}

\section{- ALARM-TRAN-CH2-SHIELD-001}

Neutron Activation Foil and Thermoluminescent Dosimeter Responses to a Polythylene Reflected Pulse of the CEA Valduc SILENE Critical Assembly, and

\section{- ALARM-TRAN-PB-SHIELD-001}

Neutron Activation Foil and Thermoluminescent Dosimeter Responses to a Lead Reflected Pulse of the CEA Valduc SILENE Critical Assembly.

\subsection{Fundamental Physics}

\section{- FUND-NCERC-PU-HE3-MULT-002}

Tungsten-Reflected Plutonium-Metal-Sphere Subcritical Measurements.

\section{Latest IRPhEP Contributions}

The 2018 edition of the IRPhEP Handbook [Ref6] is available on DVD or via direct download from the internet, and can be requested using the following website: https://www.oecd-nea.org/science/wprs/irphe/.

Since ND2016, a total of four new reactor facilities, representing 16 new experimental measurement series, have been included in the IRPhEP Handbook. The IRPhEP Handbook is divided by specific reactor types: Pressurized Water Reactors (PWR), VVER-type Reactors (VVER), Boiling Water Reactors (BWR), Liquid Metal Fast Reactors (LMFR), Gas Cooled Thermal Reactors (GCR), Gas Cooled Fast Reactors (GCFR), Light Water Moderated Reactors (LWR), Heavy Water Moderated Reactors (HWR), Molten Salt Reactors (MSR), RBMKtype Reactors (RBMK), Space Reactors (SPACE), and Fundamental Physics Reactors (FUND). The most recent contributions to the handbook include one PWR, one VVER, one LMFR, three GCFR (a reactor type previously unavailable as a benchmark on this handbook), four LWR, and six FUND benchmarks; there are no new contributions to the BWR, GCR, HWR, MSRE, RBMK, or SPACE categories. The newest evaluations are summarized:

\subsection{Pressurized Water Reactors}

\section{- VENUS-LWR-EXP-006}

Experimental Study of the VENUS Configuration No. 17.

\subsection{VVER-type Reactors}

\section{- LR(0)-VVER-RESR-003}

VVER-1000 Physics Experiments Hexagonal Lattices (1.275 cm Pitch) of Low Enriched U(3.3 wt.\% U235) $\mathrm{O}_{2}$ Fuel Assemblies in Light Water with Graphite and Fluoride Salt Insertions in Central Assembly.

\subsection{Liquid Metal Fast Reactors}

\section{- EBR2-LMFR-RESR-001}

Evaluation of Run 138B at Experimental Breeder Reactor II, A Prototypic Liquid Metal Fast Breeder Reactor.

\subsection{Gas Cooled Fast Reactors}

\section{- PROTEUS-GCFR-EXP-001}

GCFR-PROTEUS Experimental Program Core 11: Homogenous MOX Fuel Test Region,

\section{- PROTEUS-GCFR-EXP-002}

GCFR-PROTEUS Experimental Program Core 15: Homogenous MOX and Thorium Oxide Fuel Test Region, and

\section{- PROTEUS-GCFR-EXP-003}

GCFR-PROTEUS Experimental Program Core 15: MOX Lattice with Internal Thorium Metal Central Zone and $\mathrm{ThO}_{2}$ Upper Blanket.

\subsection{Light Water Moderated Reactors}

\section{- DIMPLE-LWR-EXP-003 (DRAFT)}

Light Water Moderated and Reflected Low Enriched Uranium ( 3 wt. $\%{ }^{235} \mathrm{U}$ or $7 \mathrm{wt} . \%{ }^{235} \mathrm{U}$ ) Dioxide Rod Lattices - DIMPLE CERES Phase II,

\section{- IPEN(MB01)-LWR-RESR-017}

The Inversion Point of the Isothermal Reactivity Coefficient of IPEN/MB-01 Reactor,

\section{- IPEN(MB01)-LWR-RESR-018}

Critical Loading Configurations of IPEN/MB-01 Reactor Composed of Fuel and Molybdenum Rods, and

\section{- IPEN(MB01)-LWR-RESR-019}

$\mathrm{U}(\mathrm{n}, \mathrm{f})$ and ${ }^{238} \mathrm{U}(\mathrm{n}, \gamma)$ Reaction Rates across the Fuel Pellet Radius of the IPEN/MB-01 Reactor.

\subsection{Fundamental Physics Reactors}

\section{- FCA-FUND-EXP-001}

FCA IX-7 Assembly Experiments: 20\% Enriched Uranium Metal Core Surrounded by Depleted Uranium Metal Blanket, 


\section{- ORCEF-FUND-EXP-001}

Radial Fission Rate Profiles in Aqueous Solution of $\mathrm{U}(37 \%) \mathrm{O}_{2} \mathrm{~F}_{2}$ in Spherical Geometry,

\section{- RB-FUND-EXP-008}

RB Reactor: Internal Neutron Converters (INC), Cores RB59/1983, RB60/1984 and RB61/1984,

\section{- RB-FUND-EXP-009}

RB Reactor: HERBE, Cores RB76/1990, RB77/1990 and RB77/1992,

\section{- TREAT-FUND-RESR-001}

Transient Reactor Test (TREAT) Facility: Start-up Measurements, and

\section{- TRIGA-FUND-RESR-002}

Reactor Rate Distribution Experiments at the Slovenian JSI TRIGA Mark II Research Reactor.

\subsection{Reactor Physics Uncertainty Guide}

The methods utilized by the IRPhEP to treat uncertainties encountered in experimental data and the derivation of benchmark models has historically followed guidance provided by the ICSBEP. The methods developed by the ICSBEP do not fully address the variety of reactor physics measurements and their associated uncertainties. The IRPhEP Guide to the Expression of Uncertainty has been initiated and is expected to evolve to include detailed information, and examples, regarding the evaluation of experimental data, including benchmark model development, supporting the various types of reactor physics measurements. This evaluation guide is to provide information necessary to evaluate the uncertainty typically encountered in reactor physics measurement methods. Additional information regarding the IRPhEP Uncertainty Guide is summarized elsewhere. [9]

\section{Future Work}

The annual Technical Review Group (TRG) meetings for the ICSBEP and IRPhEP were successfully held the 22-25 October 2018, and will next be held 21-25 October 2019, at the OECD NEA in Boulogne-Billancourt, France. The 2019 editions of the handbooks will be published by late
2019. Those interested in participating in these projects are invited to contact the authors.

\section{Conclusions}

Over 400 scientists from 25 different countries have combined their efforts to produce the ICSBEP and IRPhEP Handbooks. These two handbooks continue to grow and provide high-quality integral benchmark data that will be of use to the criticality safety, nuclear data, and reactor physics communities for future decades.

\section{Acknowledgments}

The ICSBEP and IRPhEP are collaborative efforts that involve numerous scientists, engineers, administrative support personnel and program sponsors from 25 different countries and the OECD NEA. The authors would like to acknowledge the efforts of all of these dedicated individuals without whom these two projects would not be possible. The reader is referred to the actual evaluations cited within the ICSBEP and IRPhEP handbooks for complete identification of the respective authors of these benchmark reports.

This paper was prepared at the Idaho National Laboratory for the U.S. Department of Energy under Contract Number (DE-AC07-05ID14517).

\section{References}

[1] J. B. Briggs, L. Scott, A. Nouri, Nucl. Sci. Eng., 145, 1-10 (2003).

[2] J. B. Briggs, J. Gulliford, Nucl. Sci. Eng., 178, 269279 (2014).

[3] J. D. Bess, J. B. Briggs, J. Gulliford, EPJ Web Conf., 146 (2017).

[4] G. Palmiotti, et al., Nucl. Sci. Eng., 178, 295-310 (2014).

[5] International Handbook of Evaluated Criticality Safety Benchmark Experiments, NEA/NSC/DOC(95)/03, OECD-NEA, Paris, France (2018).

[6] International Handbook of Evaluated Reactor Physics Benchmark Experiments, NEA/NSC/DOC(2006)1, OECD-NEA, Paris, France (2018).

[7] A. Nouri, et al., Nucl. Sci. Eng., 145, 11-19 (2003).

[8] I. Hill, N. Soppera, M. Bossant, Nucl. Sci. Eng., 178, 280-294 (2014).

[9] A. dos Santos, et al., PHYSOR 2018, Cancun, Mexico, April 22-26, 2018. 